International Journal of Pure and Applied Mathematics

Volume 86 No. 2 2013, 389-401

ISSN: 1311-8080 (printed version); ISSN: 1314-3395 (on-line version)

url: http://www.ijpam.eu

doi: http://dx.doi.org/10.12732/ijpam.v86i2.13

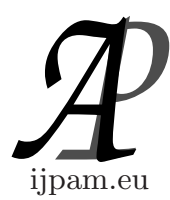

\title{
FUZZY CONNECTIONS ON UPPER SETS
}

\author{
Yong Chan Kim ${ }^{1} \S$, Jung Mi Ko ${ }^{2}$ \\ ${ }^{1,2}$ Department of Mathematics \\ Gangneung-Wonju National University \\ Gangneung, Gangwondo 210-702, KOREA
}

\begin{abstract}
In this paper, we investigate residuated (dual residuated, residuated, Galois, dual Galois) connections on upper (lower) sets in complete residuated lattices.
\end{abstract}

AMS Subject Classification: 03E72, 03G10, 06A15, 06F07

Key Words: complete residuated lattices, upper(lower) sets, isotone (antitone) maps, residuated (dual residuated, residuated, Galois, dual Galois) connections

\section{Introduction}

Hájek [7] introduced a complete residuated lattice which is an algebraic structure for many valued logic. Bělohlávek [1]-[3] developed the notion of fuzzy contexts using Galois connections with $R \in L^{X \times Y}$ on a complete residuated lattice. Georgescue and Popescu [5], [6] introduced the non-commutative fuzzy connection on generalized residuated lattice without commutative conditions. Garcia [4] investigated fuzzy connections categorically. Zhang [11], [12] introduced the notions of fuzzy completeness, join and meet for a fuzzy set. It is an important mathematical tool for algebraic structure of fuzzy contexts [1]-[3], [8]-[12].

In this paper, we investigate residuated (dual residuated, residuated, Galois, dual Galois) connections induced by various function on upper (lower) sets in

Received: February 28, 2013

(C) 2013 Academic Publications, Ltd.

${ }^{\S}$ Correspondence author

url: www.acadpubl.eu 
complete residuated lattices. We give their examples.

Definition 1.1. (see [1], [7]) An algebra $(L, \wedge, \vee, \odot, \rightarrow, 0,1)$ is called a complete residuated lattice if it satisfies the following conditions:

(C1) $L=(L, \leq, \vee, \wedge, 1,0)$ is a complete lattice with the greatest element 1 and the least element 0 ;

(C2) $(L, \odot, 1)$ is a commutative monoid;

(C3) $x \odot y \leq z$ iff $x \leq y \rightarrow z$ for $x, y, z \in L$.

In this paper, we assume $\left(L, \wedge, \vee, \odot, \rightarrow,{ }^{*} 0,1\right)$ is a complete residuated lattice with the law of double negation;i.e. $x^{* *}=x$.

Lemma 1.2. (see [1], [7]) For each $x, y, z, x_{i}, y_{i} \in L$, we have the following properties.

(1) If $y \leq z,(x \odot y) \leq(x \odot z), x \rightarrow y \leq x \rightarrow z$ and $z \rightarrow x \leq y \rightarrow x$.

(2) $x \rightarrow\left(\bigwedge_{i \in \Gamma} y_{i}\right)=\bigwedge_{i \in \Gamma}\left(x \rightarrow y_{i}\right)$.

(3) $\left(\bigvee_{i \in \Gamma} x_{i}\right) \rightarrow y=\bigwedge_{i \in \Gamma}\left(x_{i} \rightarrow y\right)$.

(4) $\bigwedge_{i \in \Gamma} y_{i}^{*}=\left(\bigvee_{i \in \Gamma} y_{i}\right)^{*}$ and $\bigvee_{i \in \Gamma} y_{i}^{*}=\left(\bigwedge_{i \in \Gamma} y_{i}\right)^{*}$.

(5) $(x \odot y) \rightarrow z=x \rightarrow(y \rightarrow z)=y \rightarrow(x \rightarrow z)$.

(6) $x \odot y=\left(x \rightarrow y^{*}\right)^{*}$ and $x \rightarrow y=y^{*} \rightarrow x^{*}$.

(7) $x \odot(x \rightarrow y) \leq y$ and $y \rightarrow z \leq x \odot y \rightarrow x \odot z$.

(8) $(x \rightarrow y) \odot(y \rightarrow z) \leq x \rightarrow z$.

(9) $x \leq y \rightarrow z$ iff $y \leq x \rightarrow z$.

(10) $y \rightarrow z \leq(x \rightarrow y) \rightarrow(x \rightarrow z)$ and $y \rightarrow z \leq(z \rightarrow x) \rightarrow(y \rightarrow x)$.

(11) $\left(\bigwedge_{i \in \Gamma} x_{i}\right) \rightarrow\left(\bigwedge_{i \in \Gamma} y_{i}\right) \geq \bigwedge_{i \in \Gamma}\left(x_{i} \rightarrow y_{i}\right)$.

(12) $\left(\bigvee_{i \in \Gamma} x_{i}\right) \rightarrow\left(\bigvee_{i \in \Gamma} y_{i}\right) \geq \bigwedge_{i \in \Gamma}\left(x_{i} \rightarrow y_{i}\right)$.

Definition 1.3. (see [1]-[3]) Let $X$ be a set. A function $e_{X}: X \times X \rightarrow L$ is called fuzzy partially order if it satisfies

(E1) reflexive if $e_{X}(x, x)=1$ for all $x \in X$,

(E2) transitive if $e_{X}(x, y) \odot e_{X}(y, z) \leq e_{X}(x, z)$, for all $x, y, z \in X$,

(E3) if $e_{X}(x, y)=e_{X}(y, x)=1$, then $x=y$.

The pair $\left(X, e_{X}\right)$ is a fuzzy partially order set (simply, fuzzy poset).

A fuzzy set $A$ is called an upper (lower) set if $A(x) \odot e_{X}(x, y) \leq A(y)$ $\left(A(x) \odot e_{X}(y, x) \leq A(y)\right)$ for all $x, y \in X . U\left(L^{X}\right)\left(D\left(L^{X}\right)\right)$ is the collection of upper (lower) sets on $L^{X}$.

Remark 1.4. (1) We define a function $e_{L^{X}}: L^{X} \times L^{X} \rightarrow L$ as $e_{L^{X}}(A, B)=$ $\bigwedge_{x \in X}(A(x) \rightarrow B(x))$. Then $\left(L^{X}, e_{L^{X}}\right)$ is a fuzzy poset from Lemma $1.2(8)$.

(2) Define a function $e_{U\left(L^{X}\right)}: U\left(L^{X}\right) \times U\left(L^{X}\right) \rightarrow L$ as $e_{U\left(L^{X}\right)}(A, B)=$ $\bigwedge_{x \in X}(A(x) \rightarrow B(x))$. Then $\left(U\left(L^{X}\right), e_{U\left(L^{X}\right)}\right)$ is a fuzzy poset. Similarly, the pair $\left(D\left(L^{X}\right), e_{D\left(L^{X}\right)}\right)$ is a fuzzy poset. 
Definition 1.5. (see [1]-[3]) Let $\left(X, e_{X}\right)$ and $\left(Y, e_{Y}\right)$ be a fuzzy poset and $f: X \rightarrow Y$ and $g: Y \rightarrow X$ maps.

(1) $\left(e_{X}, f, g, e_{Y}\right)$ is called a Galois connection if for all $x \in X, y \in Y$,

$$
e_{Y}(y, f(x))=e_{X}(x, g(y)) .
$$

(2) $\left(e_{X}, f, g, e_{Y}\right)$ is called a dual Galois connection if for all $x \in X, y \in Y$,

$$
e_{Y}(f(x), y)=e_{X}(g(y), x) .
$$

(3) $\left(e_{X}, f, g, e_{Y}\right)$ is called a residuated connection if for all $x \in X, y \in Y$,

$$
e_{Y}(f(x), y)=e_{X}(x, g(y)) .
$$

(4) $\left(e_{X}, f, g, e_{Y}\right)$ is called a dual residuated connection if for all $x \in X, y \in$ $Y$,

$$
e_{Y}(y, f(x))=e_{X}(g(y), x) .
$$

(5) A map $f:\left(X, e_{X}\right) \rightarrow\left(Y, e_{Y}\right)$ is called an isotone map if for all $x, z \in X$, $e_{X}(x, z) \leq e_{Y}(f(x), f(z))$.

(6) A map $f:\left(X, e_{X}\right) \rightarrow\left(Y, e_{Y}\right)$ is called an antitone map if for all $x, z \in X$, $e_{X}(x, z) \leq e_{Y}(f(z), f(x))$.

Definition 1.6. (see [11], [12]) Let $\left(X, e_{X}\right)$ be a fuzzy poset and $A \in L^{X}$.

(1) A point $x_{0}$ is called a join (or supremum) of $A$, denoted by $x_{0}=\sqcup A$, if it satisfies

(J1) $A(x) \leq e_{X}\left(x, x_{0}\right)$,

(J2) $\bigwedge_{x \in X}\left(A(x) \rightarrow e_{X}(x, y) \leq e_{X}\left(x_{0}, y\right)\right.$.

A point $x_{1}$ is called a meet (or infimum) of $A$, denoted by $x_{1}=\sqcap A$, if it satisfies

(M1) $A(x) \leq e_{X}\left(x_{1}, x\right)$,

(M2) $\bigwedge_{x \in X}\left(A(x) \rightarrow e_{X}(y, x) \leq e_{X}\left(y, x_{1}\right)\right.$.

The pair $\left(X, e_{X}\right)$ is called a fuzzy complete lattice if for all $A \in L^{X}, \sqcup A$ and $\sqcap A$ exist.

Let $\left(X, e_{X}\right)$ and $\left(Y, e_{Y}\right)$ be fuzzy complete lattice and $f: X \rightarrow Y$ a map.

(2) $f$ is a join preserving map if $f(\sqcup A)=\sqcup f^{\rightarrow}(A)$ for all $A \in L^{X}$, where $f^{\rightarrow}(A)(y)=\bigvee_{f(x)=y} A(x)$.

(3) $f$ is a meet preserving map if $f(\sqcap A)=\sqcap f \rightarrow(A)$ for all $A \in L^{X}$.

(4) $f$ is a meet-join map if $f(\sqcap A)=\sqcup f \rightarrow(A)$ for all $A \in L^{X}$.

(5) $f$ is a join-meet map if $f(\sqcup A)=\sqcap f^{\rightarrow}(A)$ for all $A \in L^{X}$. 
Remark 1.7. (see [11], [12]) Let $\left(X, e_{X}\right)$ be a fuzzy poset and $A \in L^{X}$.

(1) If $x_{0}$ is a join of $A$, then it is unique because $e_{X}\left(x_{0}, y\right)=e_{X}\left(y_{0}, y\right)$ for all $y \in X$, put $y=x_{0}$ or $y=y_{0}$, then $e_{X}\left(x_{0}, y_{0}\right)=e_{X}\left(y_{0}, x_{0}\right)=\top$ implies $x_{0}=y_{0}$. Similarly, if a meet of $A$ exist, then it is unique.

(2) $x_{0}$ is a join of $A$ iff $\bigwedge_{x \in X}\left(A(x) \rightarrow e_{X}(x, y)\right)=e_{X}\left(x_{0}, y\right)$.

(3) $x_{1}$ is a meet of $A$ iff $\bigwedge_{x \in X}\left(A(x) \rightarrow e_{X}(y, x)=e_{X}\left(y, x_{1}\right)\right.$.

\section{Fuzzy Connections on Upper Sets}

indent

Theorem 2.1. Let $\left(X, e_{X}\right)$ be a fuzzy poset. Then:

(1) $\left(U\left(L^{X}\right), e_{L^{X}}\right)$ is a complete.

(2) $\left(D\left(L^{X}\right), e_{L^{X}}\right)$ is a complete.

Proof. (1) For all $\mathcal{U}: U\left(L^{X}\right) \rightarrow L$, we have $\sqcup \mathcal{U}=\bigvee_{A \in U\left(L^{X}\right)} \mathcal{U}(A) \odot A$ and $\sqcap \mathcal{U}=\bigwedge_{A \in U\left(L^{X}\right)}(\mathcal{U}(A) \rightarrow A)$ from: by Lemma $1.2(5)$,

$$
\begin{aligned}
e_{U\left(L^{X}\right)}(\sqcup \mathcal{U}, B) & =\bigwedge_{A \in U\left(L^{X}\right)}\left(\mathcal{U}(A) \rightarrow e_{U\left(L^{X}\right)}(A, B)\right. \\
& =e_{U\left(L^{X}\right)}\left(\bigvee_{A \in U\left(L^{X}\right)}(\mathcal{U}(A) \odot A), B\right), \\
e_{U\left(L^{X}\right)}(B, \sqcap \mathcal{U}) & =\bigwedge_{A \in U\left(L^{X}\right)}\left(\mathcal{U}(A) \rightarrow e_{L^{X}}(B, A)\right. \\
& =e_{U\left(L^{X}\right)}\left(B, \bigwedge_{A \in U\left(L^{X}\right)}(\mathcal{U}(A) \rightarrow A)\right)
\end{aligned}
$$

Since

$$
\begin{gathered}
\bigvee_{A \in U\left(L^{X}\right)} \mathcal{U}(A) \odot A(x) \odot e_{X}(x, y) \leq \bigvee_{A \in U\left(L^{X}\right)} \mathcal{U}(A) \odot A(y) \\
\bigwedge_{A \in U\left(L^{X}\right)}(\mathcal{U}(A) \rightarrow A(x)) \odot e_{X}(x, y) \leq \bigwedge_{A \in U\left(L^{X}\right)}(\mathcal{U}(A) \rightarrow A(y)) \\
\bigvee_{A \in U\left(L^{X}\right)} \mathcal{U}(A) \odot A \in U\left(L^{X}\right) \text { and } \sqcap \mathcal{U}=\bigwedge_{A \in U\left(L^{X}\right)}(\mathcal{U}(A) \rightarrow A) \in U\left(L^{X}\right) .
\end{gathered}
$$

(2) It similarly proved as (1).

Theorem 2.2. Let $\left(X, e_{X}\right)$ and $\left(Y, e_{Y}\right)$ be fuzzy posets and $f: X \rightarrow Y$ be an isotone map. Define $f_{l} \rightarrow f_{u}: L^{X} \rightarrow L^{Y}$ as

$$
f_{l} \rightarrow(A)(y)=\bigvee_{x \in X}\left(A(x) \odot e_{Y}(y, f(x))\right),
$$




$$
f_{u}(A)(y)=\bigvee_{x \in X}\left(A(x) \odot e_{Y}(f(x), y)\right),
$$

and $f_{l}^{\leftarrow}, f_{u}^{\leftarrow}: L^{Y} \rightarrow L^{X}$ as

$$
\begin{aligned}
& f_{l}^{\leftarrow(B)(x)}=\bigvee_{z \in X}\left(B(f(z)) \odot e_{X}(x, z)\right), \\
& f_{u}^{\leftarrow}(B)(x)=\bigvee_{z \in X}\left(B(f(z)) \odot e_{X}(z, x)\right) .
\end{aligned}
$$

Then the following properties hold.

(1) $\left(D\left(L^{X}\right), f_{l} \rightarrow, f_{l}^{\leftarrow}, D\left(L^{Y}\right)\right)$ is a residuated connection and $f_{l} \rightarrow(\sqcup \mathcal{U})=$ $\sqcup\left(f_{l} \rightarrow\right) \rightarrow(\mathcal{U})$ for all $\mathcal{U} \in L^{L^{X}}$ where $\left(f_{l} \rightarrow\right) \rightarrow(\mathcal{U})(B)=\bigvee_{f_{l}(A)=B} \mathcal{U}(A)$.

(2) $\left(U\left(L^{X}\right), f_{u} \rightarrow f_{u}^{\leftarrow}, D\left(L^{X}\right)\right)$ is a residuated connection and $f_{u}(\sqcup \mathcal{U})=$ $\sqcup\left(f_{u}\right) \rightarrow(\mathcal{U})$ for all $\mathcal{U} \in L^{L^{X}}$ where $\left(f_{u}\right) \rightarrow(\mathcal{U})(B)=\bigvee_{f_{u}(A)=B} \mathcal{U}(A)$

(3) $\sqcup f_{l} \rightarrow(A) \leq f(\sqcup A)$ and $f(\sqcap A) \leq \sqcap f_{u}(A)$.

(4) $e_{L^{X}}(A, B) \leq e_{L^{Y}}\left(f_{l} \rightarrow(A), f_{l} \rightarrow(B)\right)$ and $e_{L^{X}}(A, B) \leq e_{L^{Y}}\left(f_{u}(A), f_{u} \rightarrow(B)\right)$.

(5) $e_{L^{Y}}(C, D) \leq e_{L^{X}}\left(f_{l}^{\leftarrow}(C), f_{l}^{\leftarrow}(D)\right)$ and $e_{L^{Y}}(A, B) \leq e_{L^{X}}\left(f_{u}^{\leftarrow}(C), f_{u}^{\leftarrow}(D)\right)$.

Proof. (1) Since $f_{l} \rightarrow(A)\left(y_{1}\right) \odot e_{Y}\left(y_{2}, y_{1}\right)=\bigvee_{x \in X}\left(A(x) \odot e_{Y}\left(y_{1}, f(x)\right)\right) \odot$ $e_{Y}\left(y_{2}, y_{1}\right) \leq \bigvee_{x \in X}\left(A(x) \odot e_{Y}\left(y_{2}, f(x)\right)\right)$, then $f_{l} \rightarrow(A) \in D\left(L^{Y}\right)$. Similarly, $f_{l}^{\leftarrow}(B) \in D\left(L^{X}\right)$. By Lemma $1.2(5)$, we have

$$
\begin{aligned}
e_{D\left(L^{X}\right)}\left(f_{l} \rightarrow(A), B\right) & =\bigwedge_{y \in Y}\left(f_{l}^{\rightarrow}(A)(y) \rightarrow B(y)\right) \\
& =\bigwedge_{y \in Y}\left(\bigvee_{x \in X}\left(A(x) \odot e_{Y}(y, f(x))\right) \rightarrow B(y)\right) \\
& =\bigwedge_{y \in Y} \bigwedge_{x \in X}\left(A(x) \rightarrow\left(e_{Y}(y, f(x)) \rightarrow B(y)\right)\right. \\
& =\bigwedge_{x \in X}\left(A(x) \rightarrow \bigwedge_{y \in Y}\left(e_{Y}(y, f(x)) \rightarrow B(y)\right)\right. \\
& \leq \bigwedge_{x \in X}\left(A(x) \rightarrow\left(e_{Y}(f(x), f(x)) \rightarrow B(f(x))\right)\right. \\
& =\bigwedge_{x \in X}(A(x) \rightarrow B(f(x))) \\
& \leq \bigwedge_{x \in X}\left(A(x) \rightarrow \bigvee_{z \in X}\left(B(f(z)) \odot e_{X}(x, z)\right)\right. \\
& =\bigwedge_{x \in X}\left(A(x) \rightarrow f_{l}^{\leftarrow}(B)(x)\right) \\
& =e_{D\left(L^{X}\right)}\left(A, f_{l}^{\leftarrow}(B)\right) .
\end{aligned}
$$

Let $a \leq e_{D\left(L^{X}\right)}\left(A, f_{l}^{\leftarrow}(B)\right) \leq A(x) \rightarrow f_{l}^{\leftarrow}(B)(x)$ be given. Then $a \odot A(x) \leq$ 


$$
\begin{aligned}
f_{l}^{\leftarrow}(B)(x) . & \\
a \odot f_{l} \rightarrow(A)(y) & =a \odot \bigvee_{x \in X}\left(A(x) \odot e_{Y}(y, f(x))\right. \\
& =\bigvee_{x \in X}\left(a \odot A(x) \odot e_{Y}(y, f(x))\right. \\
& \leq \bigvee_{x \in X}\left(f_{l}^{\leftarrow}(B)(x) \odot e_{Y}(y, f(x))\right) \\
& =\bigvee_{x \in X} \bigvee_{z \in X}\left(B(f(z)) \odot e_{X}(x, z) \odot e_{Y}(y, f(x))\right. \\
& \leq \bigvee_{x \in X} \bigvee_{z \in X}\left(B(f(z)) \odot e_{Y}(f(x), f(z)) \odot e_{Y}(y, f(x))\right. \\
& \leq \bigvee_{z \in X}\left(B(f(z)) \odot e_{Y}(y, f(z))\right) \\
& \leq B(y) .
\end{aligned}
$$

Hence $a \leq f_{l} \rightarrow(A)(y) \rightarrow B(y)$. Thus, $e_{D\left(L^{X}\right)}\left(A, f_{l}^{\leftarrow}(B)\right) \leq e_{D\left(L^{X}\right)}\left(f_{l} \rightarrow(A), B\right)$.

Put $B_{0}=\sqcup\left(f_{l} \rightarrow\right) \rightarrow(\mathcal{U})$. Then

$$
\begin{aligned}
e_{D\left(L^{X}\right)}\left(B_{0}, B\right) & =\bigwedge_{C \in D\left(L^{X}\right)}\left(\left(f_{l} \rightarrow\right)(\mathcal{U})(C) \rightarrow e_{D\left(L^{X}\right)}(C, B)\right) \\
& =\bigwedge_{C \in D\left(L^{X}\right)}\left(\bigvee_{f_{l} \rightarrow(A)=C} \mathcal{U}(A) \rightarrow e_{D\left(L^{X}\right)}\left(f_{l} \rightarrow(A), B\right)\right) \\
& =\bigwedge_{A \in D\left(L^{X}\right)}\left(\mathcal{U}(A) \rightarrow e_{D\left(L^{X}\right)}\left(f_{l} \rightarrow(A), B\right)\right) \\
& =\bigwedge_{A \in D\left(L^{X}\right)}\left(\mathcal{U}(A) \rightarrow e_{D\left(L^{X}\right)}\left(A, f_{l}^{\leftarrow}(B)\right)\right) \\
& =e_{D\left(L^{X}\right)}\left(\sqcup \mathcal{U}, f_{l}^{\leftarrow}(B)\right)=e_{D\left(L^{X}\right)}\left(f_{l}^{\rightarrow}(\sqcup \mathcal{U}), B\right) .
\end{aligned}
$$

Hence $B_{0}=\sqcup\left(f_{l} \rightarrow\right) \rightarrow(\mathcal{U})=f_{l} \rightarrow(\sqcup \mathcal{U})$.

(2) It is similarly proved as (1).

(3) $\sqcup f_{l} \rightarrow(A) \leq f(\sqcup A)$ and $f(\sqcap A) \leq \sqcap f_{u}(A)$ from:

$$
\begin{aligned}
e_{D\left(L^{Y}\right)}\left(\sqcup f_{l}^{\rightarrow}(A), f(\sqcup A)\right) & =\bigwedge_{y \in Y}\left(f_{l} \rightarrow(A)(y) \rightarrow e_{Y}(y, f(\sqcup A))\right. \\
& =\bigwedge_{y \in Y}\left(\bigvee _ { x \in X } \left(A(x) \odot e_{Y}(y, f(x)) \rightarrow e_{Y}(y, f(\sqcup A))\right.\right. \\
& =\bigwedge_{y \in Y} \bigwedge_{x \in X}\left(A ( x ) \rightarrow \left(e_{Y}(y, f(x))\right.\right. \\
& =\bigwedge_{x \in X}\left(A ( x ) \rightarrow \bigwedge _ { y \in Y } \left(e_{Y}(y, f(x))\right.\right. \\
& \left.=\bigwedge_{x \in X}\left(A(x) \rightarrow e_{Y}(f(x), f(\sqcup A))\right)\right) \\
& \geq \bigwedge_{x \in X}\left(A(x) \rightarrow e_{X}(x, \sqcup A)\right) \\
& =e_{X}(\sqcup A, \sqcup A)=1 . \\
& =\bigwedge_{y \in Y}\left(f_{l}(A)(y) \rightarrow e_{Y}(f(\sqcap A), y)\right) \\
& =\bigwedge_{y \in Y}\left(\bigvee_{x \in X}\left(A(x) \odot e_{Y}(f(x), y) \rightarrow e_{Y}(f(\sqcap A), y)\right)\right. \\
& =\bigwedge_{y \in Y} \bigwedge_{x \in X}\left(A(x) \rightarrow\left(e_{Y}(f(x), y) \rightarrow e_{Y}(f(\sqcap A), y)\right)\right) \\
& =\bigwedge_{x \in X}\left(A(x) \rightarrow \bigwedge_{y \in Y}\left(e_{Y}(f(x), y) \rightarrow e_{Y}(f(\sqcap A), y)\right)\right) \\
& =\bigwedge_{x \in X}\left(A(x) \rightarrow e_{Y}(f(\sqcap A), f(x))\right) \\
& \geq \bigwedge_{x \in X}\left(A(x) \rightarrow e_{X}(\sqcap A, x)\right) \\
& =e_{X}(\sqcap A, \sqcap A)=1 .
\end{aligned}
$$


(4) By Lemma 1.2(7,12), we have

$$
\begin{aligned}
& f_{l} \rightarrow(A)(y) \rightarrow f_{l} \rightarrow(B)(y) \\
& =\bigvee_{x \in X}\left(A(x) \odot e_{Y}(y, f(x))\right) \rightarrow \bigvee_{x \in X}\left(B(x) \odot e_{Y}(y, f(x))\right) \\
& \geq \bigwedge_{x \in X}\left(\left(A(x) \odot e_{Y}(y, f(x))\right) \rightarrow\left(B(x) \odot e_{Y}(y, f(x))\right)\right) \\
& \geq \bigwedge_{x \in X}(A(x) \rightarrow B(x))
\end{aligned}
$$

Hence $e_{L^{X}}(A, B) \leq e_{L^{Y}}\left(f_{l} \rightarrow(A), f_{l} \rightarrow(B)\right)$.

Other case and (5) are similarly proved.

Theorem 2.3. Let $\left(X, e_{X}\right)$ and $\left(Y, e_{Y}\right)$ be fuzzy posets and $g: X \rightarrow Y$ be a surjective map with $e_{X}(x, y)=e_{Y}(g(y), g(x))$ for each $x, y \in X$. Define $g_{l} \rightarrow g_{u}: L^{X} \rightarrow L^{Y}$ as

$$
\begin{aligned}
& g_{l}^{\rightarrow}(A)(y)=\bigwedge_{x \in X}\left(A(x) \rightarrow e_{Y}(y, g(x))\right) \\
& g_{u}^{\rightarrow}(A)(y)=\bigwedge_{x \in X}\left(A(x) \rightarrow e_{Y}(g(x), y)\right)
\end{aligned}
$$

and $g_{l}^{\leftarrow}, g_{u}^{\leftarrow}: L^{Y} \rightarrow L^{X}$ as

$$
\begin{aligned}
& g_{l}^{\leftarrow}(B)(x)=\bigwedge_{z \in X}\left(B(g(z)) \rightarrow e_{X}(x, z)\right) \\
& g_{u}^{\leftarrow}(B)(x)=\bigwedge_{z \in X}\left(B(g(z)) \rightarrow e_{X}(z, x)\right)
\end{aligned}
$$

Then the following properties hold.

(1) $\left(D\left(L^{X}\right), g_{l} \rightarrow g_{l}^{\leftarrow}, D\left(L^{Y}\right)\right)$ is a Galois connection and

$$
g_{l} \rightarrow(\sqcup \mathcal{U})=\sqcap\left(g_{l} \rightarrow\right) \rightarrow(\mathcal{U})
$$

for all $\mathcal{U} \in L^{L^{X}}$ where $\left(g_{l} \rightarrow\right) \rightarrow(\mathcal{U})(B)=\bigvee_{g_{l} \rightarrow(A)=B} \mathcal{U}(A)$.

(2) $\left(U\left(L^{X}\right), g_{u} \vec{x}, g_{u}^{\leftarrow}, U\left(L^{X}\right)\right)$ is a Galois connection and

$$
g_{u}(\sqcup \mathcal{U})=\sqcap\left(g_{u}\right) \rightarrow(\mathcal{U})
$$

for all $\mathcal{U} \in L^{L^{X}}$ where $\left(g_{\vec{u}}\right) \rightarrow(\mathcal{U})(B)=\bigvee_{g_{\vec{u}}(A)=B} \mathcal{U}(A)$.

(3) $e_{L^{X}}(A, B) \leq e_{L^{Y}}\left(g_{l} \rightarrow(B), g_{l} \rightarrow(A)\right)$ and $e_{L^{X}}(A, B) \leq e_{L^{Y}}\left(g_{u}(B), g_{u}(A)\right)$.

(4) $e_{L^{Y}}(C, D) \leq e_{L^{X}}\left(g_{l}^{\leftarrow}(D), g_{l}^{\leftarrow}(C)\right)$ and $e_{L^{Y}}(C, D) \leq e_{L^{X}}\left(g_{u}^{\leftarrow}(D), g_{u}^{\leftarrow}(C)\right)$ 
Proof. (1) Since $g_{l}(A)\left(y_{1}\right) \odot e_{Y}\left(y_{2}, y_{1}\right) \odot A(x) \leq\left(A(x) \rightarrow e_{Y}\left(y_{1}, g(x)\right)\right) \odot$ $e_{Y}\left(y_{2}, y_{1}\right) \odot A(x) \leq e_{Y}\left(y_{2}, g(x)\right)$, then $g_{l} \rightarrow(A)\left(y_{1}\right) \odot e_{Y}\left(y_{2}, y_{1}\right) \leq g_{l} \rightarrow(A)\left(y_{2}\right) ;$ i.e. $g_{l} \rightarrow(A) \in D\left(L^{Y}\right)$. Similarly, for $B \in D\left(L^{Y}\right)$, we have $g_{l}^{\leftarrow}(B) \in D\left(L^{X}\right)$.

$$
\begin{aligned}
e_{D\left(L^{Y}\right)}\left(B, g_{l}(A)\right) & =\bigwedge_{y \in Y}\left(B(y) \rightarrow g_{l}(A)(y)\right) \\
& =\bigwedge_{y \in Y}\left(B(y) \rightarrow \bigwedge_{x \in X}\left(A(x) \rightarrow e_{Y}(y, g(x))\right)\right. \\
& =\bigwedge_{y \in Y} \bigwedge_{x \in X}\left(A(x) \rightarrow\left(B(y) \rightarrow e_{Y}(y, g(x))\right)\right. \\
& =\bigwedge_{x \in X}\left(A(x) \rightarrow \bigwedge_{y \in Y}\left(B(y) \rightarrow e_{Y}(y, g(x))\right)\right. \\
& =\bigwedge_{x \in X}\left(A(x) \rightarrow \bigwedge_{z \in X}\left(B(g(z)) \rightarrow e_{Y}(g(z), g(x))\right)\right. \\
& =\bigwedge_{x \in X}\left(A(x) \rightarrow \bigwedge_{z \in X}\left(B(g(z)) \rightarrow e_{X}(x, z)\right)\right. \\
& =e_{D\left(L^{X}\right)}\left(A, g_{l}^{\leftarrow}(B)\right)
\end{aligned}
$$

Put $B_{1}=\sqcap\left(g_{l} \rightarrow\right) \rightarrow(\mathcal{U})$. Then

$$
\begin{aligned}
e_{D\left(L^{Y}\right)}\left(B, B_{0}\right) & =\bigwedge_{C \in D\left(L^{X}\right)}\left(\left(g_{l} \rightarrow\right)(\mathcal{U})(C) \rightarrow e_{D\left(L^{X}\right)}(B, C)\right) \\
& =\bigwedge_{C \in D\left(L^{X}\right)}\left(\bigvee_{g_{l} \rightarrow(A)=C}\left(\mathcal{U}(A) \rightarrow e_{D\left(L^{X}\right)}\left(B, g_{l} \rightarrow(A)\right)\right)\right. \\
& =\bigwedge_{A \in D\left(L^{X}\right)}\left(\mathcal{U}(A) \rightarrow e_{D\left(L^{X}\right)}\left(B, g_{l}(A)\right)\right) \\
& =\bigwedge_{A \in D\left(L^{X}\right)}\left(\mathcal{U}(A) \rightarrow e_{D\left(L^{X}\right)}\left(A, g_{l}^{\leftarrow}(B)\right)\right) \\
& =e_{D\left(L^{X}\right)}\left(\sqcup \mathcal{U}, g_{l}^{\leftarrow}(B)\right)=e_{D\left(L^{X}\right)}\left(B, g_{l}(\sqcup \mathcal{U})\right)
\end{aligned}
$$

Hence $B_{1}=\sqcap\left(g_{l} \rightarrow\right) \rightarrow(\mathcal{U})=g_{l} \rightarrow(\sqcup \mathcal{U})$.

(2) It is similarly proved as (1).

(3) By Lemma 1.2(10,11), we have

$$
\begin{aligned}
& g_{l} \rightarrow(B)(y) \rightarrow g_{l} \rightarrow(A)(y) \\
& =\bigwedge_{x \in X}\left(B(x) \rightarrow e_{Y}(y, g(x))\right) \rightarrow \bigwedge_{x \in X}\left(A(x) \rightarrow e_{Y}(y, g(x))\right) \\
& \geq \bigwedge_{x \in X}\left(\left(B(x) \rightarrow e_{Y}(y, g(x))\right) \rightarrow\left(A(x) \rightarrow e_{Y}(y, g(x))\right)\right) \\
& \geq \bigwedge_{x \in X}(A(x) \rightarrow B(x))
\end{aligned}
$$

Other case and (4) are similarly proved.

Theorem 2.4. Let $\left(X, e_{X}\right)$ and $\left(Y, e_{Y}\right)$ be fuzzy posets and $h: X \rightarrow Y$ be a surjective map with $e_{X}(x, y)=e_{Y}(h(y), h(x))$ for each $x, y \in X$. Define $h_{l}, h_{u}: L^{X} \rightarrow L^{Y}$ as

$$
\begin{aligned}
& h_{l}(A)(y)=\bigvee_{x \in X}\left(A^{*}(x) \odot e_{Y}(y, h(x))\right), \\
& h_{u}^{\rightarrow}(A)(y)=\bigvee_{x \in X}\left(A^{*}(x) \odot e_{Y}(h(x), y)\right),
\end{aligned}
$$


and $h_{l}^{\leftarrow}, h_{u}^{\leftarrow}: L^{Y} \rightarrow L^{X}$ as

$$
\begin{aligned}
& h_{l}^{\leftarrow}(B)(x)=\bigvee_{z \in X}\left(B^{*}(h(z)) \odot e_{X}(x, z)\right), \\
& h_{u}^{\leftarrow}(B)(x)=\bigvee_{z \in X}\left(B^{*}(h(z)) \odot e_{X}(z, x)\right)
\end{aligned}
$$

Then the following properties hold.

(1) $\left(D\left(L^{X}\right), h_{l} \rightarrow h_{l}^{\leftarrow}, D\left(L^{Y}\right)\right)$ is a dual Galois connection and $h_{l} \rightarrow(\sqcap \mathcal{U})=$ $\sqcup\left(h_{l} \rightarrow\right) \rightarrow(\mathcal{U})$ for all $\mathcal{U} \in L^{L^{X}}$ where $\left(h_{l} \rightarrow\right)(\mathcal{U})(B)=\bigvee_{h_{l} \rightarrow(A)=B} \mathcal{U}(A)$.

(2) $\left(U\left(L^{X}\right), h_{u}, h_{u}^{\leftarrow}, U\left(L^{X}\right)\right)$ is a dual Galois connection and $h_{u}(\sqcap \mathcal{U})=$

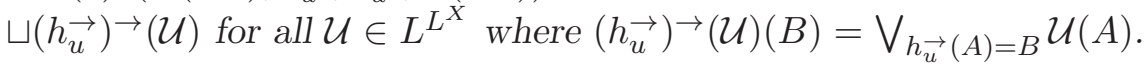

$(3) e_{L^{X}}(A, B) \leq e_{L^{Y}}\left(h_{l} \rightarrow(B), h_{l} \rightarrow(A)\right)$ and $e_{L^{X}}(A, B) \leq e_{L^{Y}}\left(h_{u}(B), h_{u}(A)\right)$.

(4) $e_{L^{Y}}(C, D) \leq e_{L^{X}}\left(h_{l}^{\leftarrow}(D), h_{l}^{\leftarrow}(C)\right)$ and $e_{L^{Y}}(C, D) \leq e_{L^{X}}\left(h_{u}^{\leftarrow}(D), h_{u}^{\leftarrow}(C)\right)$

Proof. (1) For $A \in D\left(L^{X}\right)$, since

$$
\begin{aligned}
& h_{l} \rightarrow(A)\left(y_{1}\right) \odot e_{Y}\left(y_{2}, y_{1}\right) \\
& =\bigvee_{x \in X}\left(A^{*}(x) \odot e_{Y}\left(y_{1}, f(x)\right)\right) \odot e_{Y}\left(y_{2}, y_{1}\right) \\
& \leq \bigvee_{x \in X}\left(A^{*}(x) \odot e_{Y}\left(y_{2}, f(x)\right)\right)
\end{aligned}
$$

then $h_{l} \rightarrow(A) \in D\left(L^{Y}\right)$. Similarly, for $B \in D\left(L^{Y}\right)$, we have $h_{l}^{\leftarrow}(B) \in D\left(L^{X}\right)$.

$$
\begin{aligned}
e_{D\left(L^{X}\right)}\left(h_{l} \rightarrow(A), B\right) & =\bigwedge_{y \in Y}\left(h_{l}(A)(y) \rightarrow B(y)\right) \\
& =\bigwedge_{y \in Y}\left(\bigvee_{x \in X}\left(A^{*}(x) \odot e_{Y}(y, h(x))\right) \rightarrow B(y)\right) \\
& =\bigwedge_{y \in Y} \bigwedge_{x \in X}\left(e_{Y}(y, h(x)) \rightarrow\left(A^{*}(x) \rightarrow B(y)\right)\right. \\
& =\bigwedge_{y \in Y} \bigwedge_{x \in X}\left(e_{Y}(y, h(x)) \rightarrow\left(B^{*}(y) \rightarrow A(x)\right)\right. \\
& =\bigwedge_{y \in Y} \bigwedge_{x \in X}\left(e_{Y}(y, h(x)) \odot B^{*}(y) \rightarrow A(x)\right) \\
& \leq \bigwedge_{x \in X}\left(\bigvee_{z \in X} e_{Y}(h(z), h(x)) \odot B^{*}(h(z)) \rightarrow A(x)\right) \\
& \leq \bigwedge_{x \in X}\left(\bigvee_{z \in X} e_{X}(x, z) \odot B^{*}(h(z)) \rightarrow A(x)\right) \\
& =e_{D\left(L^{X}\right)}\left(h_{l}^{\leftarrow}(B), A\right) \\
e_{D\left(L^{X}\right)}\left(h_{l}^{\leftarrow}(B), A\right) & =\bigwedge_{x \in X}\left(h_{l}(B)(x) \rightarrow A(x)\right) \\
& =\bigwedge_{x \in X}\left(\bigvee_{z \in X}\left(B^{*}(h(z)) \odot e_{X}(x, z)\right) \rightarrow A(x)\right) \\
& =\bigwedge_{x \in X} \bigwedge_{z \in X}\left(e_{X}(x, z) \rightarrow\left(B^{*}(h(z)) \rightarrow A(x)\right)\right. \\
& =\bigwedge_{x \in X} \bigwedge_{z \in X}\left(e_{X}(x, z) \rightarrow(A(x) \rightarrow B(h(z)))\right. \\
& =\bigwedge_{x \in X} \bigwedge_{z \in X}\left(e_{X}(x, z) \odot A^{*}(x) \rightarrow B(h(z))\right) \\
& =\bigwedge_{x \in X} \bigwedge_{z \in X}\left(e_{Y}(h(z), h(x)) \odot A^{*}(x) \rightarrow B(h(z))\right) \\
& =e_{D\left(L^{X}\right)}\left(h_{l}^{\leftarrow}(B), A\right)
\end{aligned}
$$


Put $B_{0}=\sqcup\left(h_{l} \rightarrow\right) \rightarrow(\mathcal{U})$. Then

$$
\begin{aligned}
e_{D\left(L^{X}\right)}\left(B_{0}, B\right) & =\bigwedge_{C \in D\left(L^{X}\right)}\left(\left(h_{l} \rightarrow \rightarrow(\mathcal{U})(C) \rightarrow e_{D\left(L^{X}\right)}(C, B)\right)\right. \\
& =\bigwedge_{C \in D\left(L^{X}\right)}\left(\bigvee_{h_{l} \rightarrow(A)=C}\left(\mathcal{U}(A) \rightarrow e_{D\left(L^{X}\right)}\left(h_{l} \rightarrow(A), B\right)\right)\right. \\
& =\bigwedge_{A \in D\left(L^{X}\right)}\left(\mathcal{U}(A) \rightarrow e_{D\left(L^{X}\right)}\left(h_{l} \rightarrow(A), B\right)\right) \\
& =\bigwedge_{A \in D\left(L^{X}\right)}\left(\mathcal{U}(A) \rightarrow e_{U\left(L^{X}\right)}\left(h_{l}^{\leftarrow}(B), A\right)\right) \\
& =e_{D\left(L^{X}\right)}\left(h_{l}^{\leftarrow}(B), \sqcap \mathcal{U}\right)=e_{U\left(L^{X}\right)}\left(h_{l}(\sqcap \mathcal{U}), B\right)
\end{aligned}
$$

Hence $B_{0}=\sqcup\left(h_{l} \rightarrow\right) \rightarrow(\mathcal{U})=h_{l} \rightarrow(\sqcap \mathcal{U})$.

(2) It is similarly proved as (1).

(3) By Lemma 1.2(6,12), we have

$$
\begin{aligned}
& h_{l}(B)(y) \rightarrow h_{l} \rightarrow(A)(y) \\
& =\bigvee_{x \in X}\left(B^{*}(x) \odot e_{Y}(y, h(x))\right) \rightarrow \bigvee_{x \in X}\left(A^{*}(x) \odot e_{Y}(y, g(x))\right) \\
& \geq \bigwedge_{x \in X}\left(\left(B^{*}(x) \odot e_{Y}(y, g(x))\right) \rightarrow\left(A^{*}(x) \odot e_{Y}(y, g(x))\right)\right) \\
& \geq \bigwedge_{x \in X}\left(B^{*}(x) \rightarrow A^{*}(x)\right)=\bigwedge_{x \in X}(A(x) \rightarrow B(x))
\end{aligned}
$$

Other case and (4) are similarly proved.

Theorem 2.5. Let $\left(X, e_{X}\right)$ and $\left(Y, e_{Y}\right)$ be fuzzy posets and $k: X \rightarrow Y$ be a surjective map with $e_{X}(x, y)=e_{Y}(k(y), k(x))$ for each $x, y \in X$. Define $k_{l} \rightarrow, k_{u}: L^{X} \rightarrow L^{Y}$ as

$$
\begin{aligned}
& k_{l}^{\rightarrow}(A)(y)=\bigwedge_{x \in X}\left(e_{Y}(y, f(x)) \rightarrow A(x)\right), \\
& k_{u}^{\rightarrow}(A)(y)=\bigwedge_{x \in X}\left(e_{Y}(f(x), y) \rightarrow A(x)\right),
\end{aligned}
$$

and $k_{l}^{\leftarrow}, k_{u}^{\leftarrow}: L^{Y} \rightarrow L^{X}$ as

$$
\begin{aligned}
& k_{l}^{\leftarrow}(B)(x)=\bigvee_{z \in X}\left(B(f(z)) \odot e_{X}(x, z)\right), \\
& k_{u}^{\leftarrow}(B)(x)=\bigvee_{z \in X}\left(B(f(z)) \odot e_{X}(z, x)\right) .
\end{aligned}
$$

Then the following properties hold.

(1) $\left(D\left(L^{X}\right), k_{l} \rightarrow, k_{l}^{\leftarrow}, U\left(L^{Y}\right)\right)$ is a dual residuated connection and $k_{l} \rightarrow(\sqcap \mathcal{U})=$ $\sqcap\left(k_{l} \rightarrow\right) \rightarrow(\mathcal{U})$ for all $\mathcal{U} \in L^{L^{X}}$ where $\left(k_{l} \rightarrow\right) \rightarrow(\mathcal{U})(B)=\bigvee_{k_{l} \rightarrow(A)=B} \mathcal{U}(A)$ 
(2) $\left(U\left(L^{X}\right), k_{u}^{\rightarrow}, k_{u}^{\leftarrow}, D\left(L^{Y}\right)\right)$ is a dual residuated connection and $k_{u} \rightarrow(\sqcap \mathcal{U})=$ $\sqcap\left(k_{u}\right) \rightarrow(\mathcal{U})$ for all $\mathcal{U} \in L^{L^{X}}$ where $\left(k_{u}\right) \rightarrow(\mathcal{U})(B)=\bigvee_{k_{u}(A)=B} \mathcal{U}(A)$.

$(3) e_{L^{X}}(A, B) \leq e_{L^{Y}}\left(f_{l} \rightarrow(A), f_{l} \rightarrow(B)\right)$ and $e_{L^{X}}(A, B) \leq e_{L^{Y}}\left(f_{u}(A), f_{u} \rightarrow(B)\right)$.

(4) $e_{L^{Y}}(C, D) \leq e_{L^{X}}\left(f_{l}^{\leftarrow}(C), f_{l}^{\leftarrow}(D)\right)$ and $e_{L^{Y}}(A, B) \leq e_{L^{X}}\left(f_{u}(C), f_{u}^{\leftarrow}(D)\right)$

Proof. (1) Since $k_{l} \rightarrow(A)\left(y_{1}\right) \odot e_{Y}\left(y_{1}, y_{2}\right) \odot e_{Y}\left(y_{2}, f(x)\right) \leq\left(e_{Y}\left(y_{1}, f(x)\right) \rightarrow\right.$ $A(x)) \odot e_{Y}\left(y_{1}, f(x)\right) \leq A(x)$ iff $k_{l} \rightarrow(A)\left(y_{1}\right) \odot e_{Y}\left(y_{1}, y_{2}\right) \leq k_{l}(A)\left(y_{2}\right)$, then $k_{l} \rightarrow(A) \in U\left(L^{Y}\right)$. Similarly, $k_{l}^{\leftarrow}(B) \in D\left(L^{X}\right)$.

$$
\begin{aligned}
e_{D\left(L^{X}\right)}\left(B, k_{l} \rightarrow(A)\right) & =\bigwedge_{y \in Y}\left(B(y) \rightarrow k_{l}^{\rightarrow}(A)(y)\right) \\
& =\bigwedge_{y \in Y}\left(B(y) \rightarrow \bigwedge_{x \in X}\left(e_{Y}(y, k(x)) \rightarrow A(x)\right)\right. \\
& =\bigwedge_{y \in Y} \bigwedge_{x \in X}\left(B(y) \odot e_{Y}(y, k(x)) \rightarrow A(x)\right) \\
& =\bigwedge_{x \in X}\left(\bigvee_{y \in Y} B(y) \odot e_{Y}(y, k(x)) \rightarrow A(x)\right) \\
& =\bigwedge_{x \in X}\left(\bigvee_{z \in X} B(k(z)) \odot e_{Y}(k(z), k(x)) \rightarrow A(x)\right) \\
& =\bigwedge_{x \in X}\left(\bigvee_{z \in X} B(k(z)) \odot e_{X}(x, z) \rightarrow A(x)\right) \\
& =e_{U\left(L^{X}\right)}\left(k_{l}^{\leftarrow}(B), A\right)
\end{aligned}
$$

Put $B_{1}=\sqcap\left(k_{l} \rightarrow\right) \rightarrow(\mathcal{U})$. Then

$$
\begin{aligned}
e_{U\left(L^{Y}\right)}\left(B, B_{1}\right) & =\bigwedge_{C \in U\left(L^{Y}\right)}\left(\left(k_{l} \rightarrow\right)(\mathcal{U})(C) \rightarrow e_{U\left(L^{Y}\right)}(B, C)\right) \\
& =\bigwedge_{C \in U\left(L^{Y}\right)}\left(\bigvee_{g_{l} \rightarrow(A)=C}\left(\mathcal{U}(A) \rightarrow e_{U\left(L^{Y}\right)}\left(B, k_{l} \rightarrow(A)\right)\right)\right. \\
& =\bigwedge_{A \in D\left(L^{X}\right)}\left(\mathcal{U}(A) \rightarrow e_{U\left(L^{Y}\right)}\left(B, k_{l} \rightarrow(A)\right)\right) \\
& =\bigwedge_{A \in D\left(L^{X}\right)}\left(\mathcal{U}(A) \rightarrow e_{D\left(L^{X}\right)}\left(k_{l}^{\leftarrow}(B), A\right)\right) \\
& =e_{D\left(L^{X}\right)}\left(k_{l}^{\leftarrow}(B), \sqcap \mathcal{U}\right)=e_{U\left(L^{Y}\right)}\left(B, k_{l} \rightarrow(\sqcap \mathcal{U})\right)
\end{aligned}
$$

Hence $B_{1}=\sqcap\left(k_{l} \rightarrow\right) \rightarrow(\mathcal{U})=k_{l} \rightarrow(\sqcap \mathcal{U})$.

(2) It is similarly proved as (1).

(3) By Lemma 1.2(10,11), we have

$$
\begin{aligned}
& k_{l} \rightarrow(A)(y) \rightarrow k_{l} \rightarrow(B)(y) \\
& \left.=\bigwedge_{x \in X}\left(e_{Y}(y, f(x)) \rightarrow A(x)\right) \rightarrow \bigwedge_{x \in X}\left(e_{Y}(y, f(x)) \rightarrow B(x)\right)\right) \\
& \left.\geq \bigwedge_{x \in X}\left(\left(e_{Y}(y, f(x)) \rightarrow A(x)\right) \rightarrow\left(e_{Y}(y, f(x)) \rightarrow B(x)\right)\right)\right) \\
& \geq \bigwedge_{x \in X}(A(x) \rightarrow B(x))
\end{aligned}
$$

Other case and (4) are similarly proved. 
Example 2.6. Define a binary operation $\odot$ (called Łukasiewicz conjection) on $L=[0,1]$ by

$$
x \odot y=\max \{0, x+y-1\}, x \rightarrow y=\min \{1-x+y, 1\} .
$$

(1) Let $\left(X=\{a, b, c\}, e_{X}\right)$ and $\left(Y=\{x, y, z\}, e_{Y}\right)$ be a fuzzy poset with $e_{X}=\left(e_{X}(a, b)\right)$ and $e_{Y}=\left(e_{Y}(x, y)\right)$ as follows:

$$
e_{X}=\left(\begin{array}{lll}
1.0 & 0.7 & 0.4 \\
0.3 & 1.0 & 0.6 \\
0.5 & 0.5 & 1.0
\end{array}\right) \quad e_{Y}=\left(\begin{array}{lll}
1.0 & 0.8 & 0.6 \\
0.6 & 1.0 & 0.5 \\
0.7 & 0.6 & 1.0
\end{array}\right)
$$

We define $f: X \rightarrow Y$ with $f(a)=x, f(b)=f(c)=y$. Then $f$ is an isotone map. It satisfies the conditions of Theorem 2.2. Thus, the properties of Theorem hold.

(2) Let $\left(X=\{a, b, c\}, e_{X}\right)$ and $\left(Y=\{x, y, z\}, e_{Y}\right)$ be a fuzzy poset with $e_{X}=\left(e_{X}(a, b)\right)$ and $e_{Y}=\left(e_{Y}(x, y)\right)$ as follows:

$$
e_{X}=\left(\begin{array}{lll}
1.0 & 0.8 & 0.6 \\
0.6 & 1.0 & 0.5 \\
0.7 & 0.6 & 1.0
\end{array}\right) \quad e_{Y}=\left(\begin{array}{lll}
1.0 & 0.8 & 0.6 \\
0.6 & 1.0 & 0.7 \\
0.5 & 0.6 & 1.0
\end{array}\right)
$$

We define $g: X \rightarrow Y$ with $g(a)=y, g(b)=x, g(c)=z$. Then $e_{X}(a, b)=$ $e_{Y}(g(b), g(a))$ for all $a, b \in X$. It satisfies the conditions of Theorems 2.3, 2.4 and 2.5. Thus, the properties of Theorems 2.3, 2.4 and 2.5 hold.

\section{References}

[1] R. Bělohlávek, Fuzzy Galois connections, Math. Log. Quart., 45 (1999), 497-504.

[2] R. Bělohlávek, Concept lattices and order in fuzzy logic, Ann. Pure Appl. Logic, 128(2004), 277-298.

[3] R. Bělohlávek, Lattices of fixed points of Galois connections, Math. Logic Quart., 47 (2001), 111-116.

[4] J.G. Garcia, I.M. Perez, M.A. Vicente, D. Zhang, Fuzzy Galois connections categorically, Math. Log. Quart., 56(2010), 131-147. 
[5] G. Georgescu, A. Popescue, Non-commutative Galois connections, Soft Computing, 7(2003), 458-467.

[6] G. Georgescu, A. Popescue, Non-dual fuzzy connections, Arch. Math. Log. 43(2004), 1009-1039.

[7] P. Hájek, Metamathematices of Fuzzy Logic, Kluwer Academic Publishers, Dordrecht (1998).

[8] H. Lai, D. Zhang, Complete and directed complete $\Omega$-categories, Theoretical Computer Science, 388 (2007), 1-25.

[9] H. Lai, D. Zhang, Concept lattices of fuzzy contexts, Int. J. Approx. Reasoning, 50(2009), 695-707.

[10] W. Yao, L.X. Lu, Fuzzy Galois connections on fuzzy posets, Math. Log. Quart., 55(2009), 105-112.

[11] Q. Y. Zhang, L. Fan, Continuity in quantitive domains, Fuzzy Sets and Systems, 154(2005), 118-131.

[12] Q. Y. Zhang, W. X. Xie, Fuzzy complete lattices, Fuzzy Sets and Systems, 160 (2009), 2275-2291. 
